\title{
Singlet and triplet excited-state dynamics of a nonfullerene electron acceptor Y6
}

AUTHOR NAMES;

Shin-ichiro Natsuda ${ }^{1}$, Yuji Sakamoto ${ }^{1}$, Taiki Takeyama ${ }^{1}$, Rei Shirouchi ${ }^{1}$, Toshiharu Saito ${ }^{1}$, Yasunari Tamai ${ }^{1,2 *}$, Hideo Ohkita ${ }^{l}$

AUTHOR ADDRESS;

'Department of Polymer Chemistry, Graduate School of Engineering, Kyoto University, Katsura, Nishikyo, Kyoto 615-8510, Japan

¥apan Science and Technology Agency (JST), PRESTO, 4-1-8 Honcho Kawaguchi, Saitama 3320012, Japan

\section{AUTHOR INFORMATION}

Corresponding Author*E-mail: tamai@photo.polym.kyoto-u.ac.jp 


\section{ABSTRACT}

Understanding the excited-state dynamics of nonfullerene electron acceptors is essential for further improvement of organic solar cells. Herein, we investigated the singlet and triplet excited-state dynamics in Y6, a novel nonfullerene acceptor, using transient absorption spectroscopy. We found that pristine Y6 films show biphasic singlet exciton decay kinetics with decay constants of $\sim 220$ ps and $\sim 1200 \mathrm{ps}$, which is the origin of the large discrepancies in the previously reported exciton lifetimes in the solid state. The majority of the Y6 singlet excitons decayed with the faster $(\sim 220$ ps) component, whereas a clear photoluminescence with the slower ( $\sim 1200 \mathrm{ps})$ component was observed. Y6 singlet excitons undergo fast diffusion in the crystalline domains, resulting in fast singlet-singlet exciton annihilation, after which ultrafast triplet formation, assigned to singlet fission from higher excited singlet states, is observed.

\section{TOC GRAPHICS}

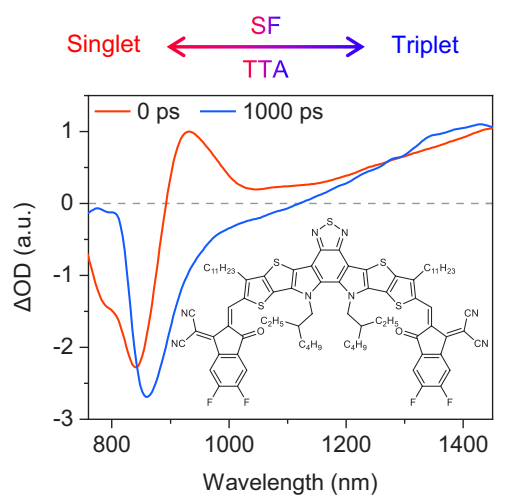

KEYWORDS: exciton diffusion, organic solar cell, photon upconversion, singlet-singlet exciton annihilation, singlet fission, transient absorption spectroscopy 
The power conversion efficiencies (PCEs) of organic solar cells (OSCs) now exceed $18 \%,{ }^{1-3}$ largely owing to the development of novel nonfullerene acceptors (NFAs). ${ }^{4-12}$ OSCs consisting of a low-bandgap NFA and a wide-bandgap donor polymer exhibit strong and complementary absorption in the visible to near-IR region, resulting in efficient photocurrent generation comparable to their inorganic counterparts. Acceptor singlet excitons are photogenerated in these OSCs after exposure to near-IR light, which is in sharp contrast to fullerene-based OSCs, wherein singlet excitons are predominantly generated in the donor polymers. Hence, understanding the excited-state dynamics of NFAs is of critical importance for NFA-based OSCs.

State-of-the-art OSCs often use Y6 (the chemical structure, steady-state absorption, and photoluminescence (PL) spectra of Y6 are found in the Supporting Information) and its derivatives as NFAs. These NFAs are strongly crystalline and exhibit deep highest occupied molecular orbital (HOMO) energy levels, large absorption cross-sections in the near-IR region, and optical bandgaps suitable for solar cell applications. Therefore, a lot of studies on Y6-based OSCs have been conducted. ${ }^{13-19}$ In contrast, the excited-state properties of Y6 are still not fully understood. For example, the singlet exciton diffusion length $L_{\mathrm{D}}$, which is defined as $L_{\mathrm{D}}=(D \tau)^{0.5}$, where $D$ is the exciton diffusion constant and $\tau$ is the exciton lifetime, is an important parameter that affects the PCE. Nevertheless, the reported $\tau$ values for Y6 thin films cover a significant range, from $\sim 50 \mathrm{ps}$ to $\sim 1.8$ ns. ${ }^{20-25}$ Therefore, further research is necessary to understand the origins of such large discrepancies as well as reliable excited-state properties. Alongside singlet excited-state properties, those of triplet excited states are also important because they affect the charge recombination dynamics in OSCs. ${ }^{26,27}$ 
Herein, we study the singlet and triplet excited-state dynamics of Y6 in solution and solid states using transient absorption (TA) spectroscopy. We found that pristine Y6 films show biphasic singlet exciton decay kinetics with decay constants of $\sim 220$ ps and $\sim 1200$ ps, which is the origin of the large discrepancies in the previously reported exciton lifetimes in the solid state. Y6 shows a relatively fast exciton diffusion constant and a small energy difference between the lowest excited singlet and triplet states $\left(\mathrm{S}_{1}\right.$ and $\mathrm{T}_{1}$ states $)$. Ultrafast triplet exciton formation was observed at a high excitation fluence, which is attributable to singlet fission from higher excited singlet states $\left(\mathrm{S}_{\mathrm{n}}\right.$ states) following singlet-singlet exciton annihilation (SSA).

Singlet excited states of Y6 in the solid state. Figure 1a shows the TA spectra of a pristine Y6 film (70 $\mathrm{nm}$ thick). A photoinduced absorption (PIA) band at $\sim 930 \mathrm{~nm}$ and a broad tail above $1100 \mathrm{~nm}$ appeared immediately after photoexcitation at $800 \mathrm{~nm}$, as in the case of Y6 in a chloroform (CF) solution (Figure S3). These bands are assigned to Y6 singlet excitons in the solid state. ${ }^{28}$ In addition, a sharp negative signal was observed at $\sim 850 \mathrm{~nm}$, which is attributable to the ground-state bleaching (GSB) of Y6. In TA measurements, the bimolecular exciton deactivation process due to SSA generates an additional decay channel that leads to faster exciton decay. ${ }^{29,30}$ Hence, SSA must be avoided when the intrinsic singlet exciton decay kinetics is observed. Because it is difficult to avoid SSA in the thin Y6 film, which, as discussed later, is a sign of fast exciton diffusion, we performed TA measurements for a 240-nm-thick film (the TA spectra of the thick Y6 film are presented in Figure S6) with weak excitation fluences. The excitation wavelength was set to 400 $\mathrm{nm}$, where the absorption cross-section of Y6 is approximately six times smaller than that at the peak wavelength, to keep the exciton density low. Figure $1 \mathrm{~b}$ shows the excitation-fluence dependence of the exciton decay kinetics monitored at $930 \mathrm{~nm}$. The decay kinetics are independent of the excitation fluence, at least below $3.0 \mu \mathrm{J} \mathrm{cm}^{-2}$; however, decay was slightly faster at $6.4 \mu \mathrm{J}$ 
$\mathrm{cm}^{-2}$ owing to SSA. We simultaneously fitted the two fluences $\left(3.0 \mu \mathrm{J} \mathrm{cm} \mathrm{cm}^{-2}\right.$ and $\left.2.2 \mu \mathrm{J} \mathrm{cm}^{-2}\right)$ with a shared time constant. The exciton decay was well fitted by an exponential function with a lifetime of $\sim 220 \mathrm{ps}$. Note that the decay kinetics of the broad PIA tail above $1100 \mathrm{~nm}$ on early times were also well reproduced by the same exponential function.
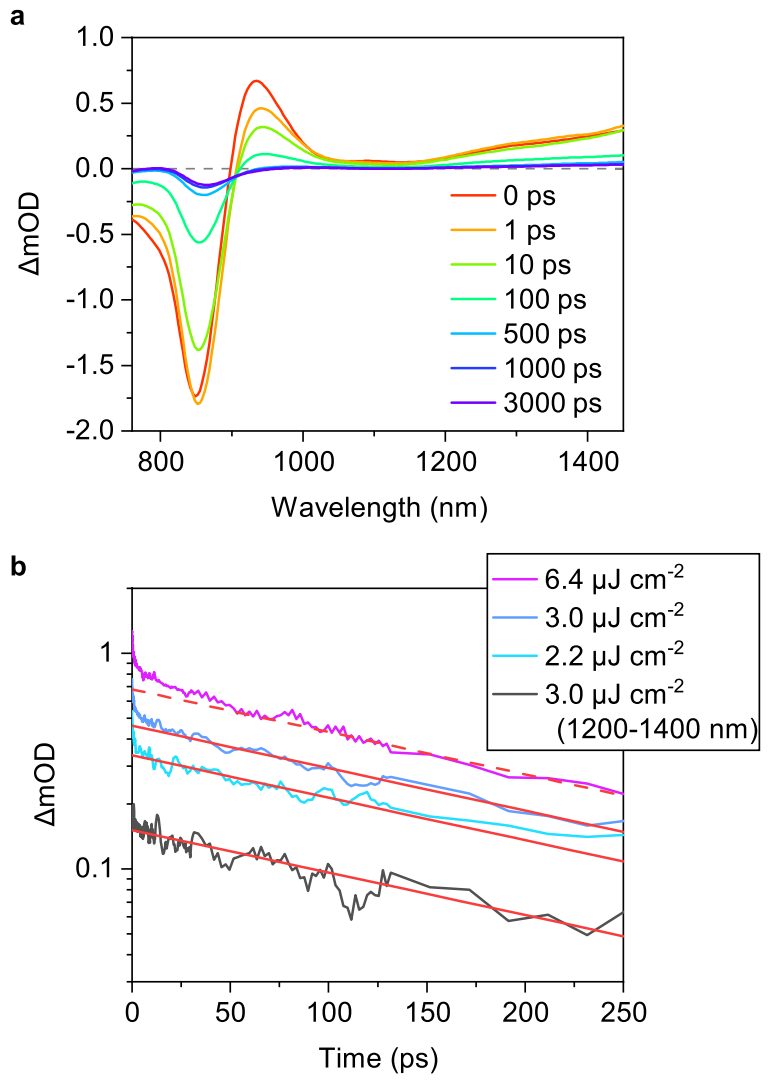

Figure 1. (a) TA spectra of a pristine Y6 film (70 $\mathrm{nm}$ thick). The excitation wavelength was set to $800 \mathrm{~nm}$ with a fluence of $3.8 \mu \mathrm{J} \mathrm{cm}^{-2}$. (b) Time evolution of TA signals monitored at 930 $\mathrm{nm}$ at various excitation fluences (colored lines). The excitation wavelength was $400 \mathrm{~nm}$. A 240-nm-thick pristine Y6 film was used to avoid SSA. The black line represents the decay kinetics of the TA signals averaged over the 1200-1400 nm region. The red lines represent the best fitted exponential curves. 
Interestingly, the singlet exciton lifetime measured by TA spectroscopy is significantly shorter than the PL decay lifetime obtained by the time-correlated single-photon-counting (TCSPC) method ( 1.2 ns, Figure S2b). As mentioned above, this large discrepancy has also been reported previously: exciton lifetimes of a few hundreds of picoseconds were obtained by TA measurements, whereas lifetimes of $>1$ ns were obtained by time-resolved PL (TRPL) measurements. ${ }^{21-25}$ Close inspection of the TA spectra (Figures 1a and S6a) reveals that the TA signal in the 900-950 nm region turned into negative at later times, which is attributable to the stimulated emission (SE) of Y6 because its spectral position coincides with the PL spectrum (Figure S1b). This indicates that SE with a longer time constant can also be observed in the TA spectra, even though the singlet exciton absorption had already disappeared. Interestingly, a certain portion of the GSB signal was still observed on the nanosecond time scale, indicating that long-lived transient species were generated, which we attribute to triplet excitons, as described later (Figure 3). Therefore, we speculate that the PL on the nanosecond time scale may be induced by the $\mathrm{S}_{1}$ state repopulated through triplet-triplet exciton annihilation (TTA). As shown in Figure S6, Y6 triplet excitons were generated in the pristine Y6 films, which, then, decayed on the nanosecond time scale, suggesting the presence of TTA because decay on nanosecond time scale is too fast to be assigned to intrinsic deactivation of triplet excitons generated in organic molecules without heavy metals. Another possible explanation for the slow PL may be the presence of highly ordered regions with a suppressed nonradiative decay rate in Y6 films. This is because (TR)PL measurements are dominated by emissive states, whereas TA measurements can directly probe the exciton density. This speculation originates from the fact that the peak positions of the GSB red-shift with time, suggesting the presence of Y6 excitons in highly ordered regions, where they are expected to decay more slowly with higher PL quantum yields. Further studies are required to reveal the origin of 
the slow PL. Nevertheless, what is important here is that the majority of the singlet excitons decay with a time constant of 220 ps. Therefore, we chose 220 ps as the exciton lifetime of Y6 in the solid state for later discussion. Triplet excitons generation in pristine Y6 films will be further discussed in the later section.

Singlet exciton diffusion. The diffusion properties of Y6 singlet excitons can be determined by analyzing the excitation-fluence-dependent kinetics of exciton decay. ${ }^{29-31}$ The rate equation for singlet excitons, including SSA, is given by

$$
\frac{\mathrm{d} n_{\mathrm{S}}(t)}{\mathrm{d} t}=-k n_{\mathrm{S}}(t)-\frac{1}{2} \gamma(t) n_{\mathrm{S}}(t)^{2}
$$

where $n_{\mathrm{s}}(t), k$, and $\gamma(t)$ are the exciton density at time $t$ after photoexcitation, the rate constant for monomolecular deactivation given by the inverse of the exciton lifetime ( 220 ps for Y6 in the solid state), and the SSA rate coefficient, respectively (details are found in the Supporting Information). Equation 1 can be analytically solved as

$$
\begin{gathered}
n_{\mathrm{S}}(t)=\frac{n_{0} \exp (-k t)}{1+\frac{n_{0}}{2} G(t)} \\
G(t)=8 \pi D R\left[\frac{1}{k}\left(1-e^{-k t}\right)+\frac{R}{\sqrt{2 D k}} \operatorname{erf}(\sqrt{k t})\right]
\end{gathered}
$$

where $n_{0}$ is the initial exciton density, and "erf" denotes the error function. Herein, we used a threedimensionally (3D) isotropic diffusion model, ${ }^{29-31}$ and the effective reaction radius $R$ is assumed to be $1 \mathrm{~nm}$ as a typical value for small molecules to provide a straightforward comparison with previous studies. 
Singlet excitons decay faster with increasing excitation fluence in the 70-nm-thick Y6 film due to SSA. The red and blue lines in Figure 2 are best fits obtained using Equations 2 and 3 with and without the second term (the error function term) in Equation 3, respectively. Both fitting curves reproduce the experimentally observed exciton decay kinetics well, which is a sign of a relatively fast diffusion because the error function term is negligible when $t \gg>R^{2} /(2 \pi D)$. From the fitting with the error function term (red line), $D$ is evaluated to be $2.1 \times 10^{-2} \mathrm{~cm}^{2} \mathrm{~s}^{-1}$. This value is approximately one order of magnitude larger than those of typical conjugated polymers ${ }^{31}$ but similar to those of recent novel NFAs. ${ }^{21,32} L_{\mathrm{D}}$ was calculated to be $\sim 21 \mathrm{~nm}$; this relatively long diffusion length is a key factor that determines efficient charge generation in Y6-based OSCs.

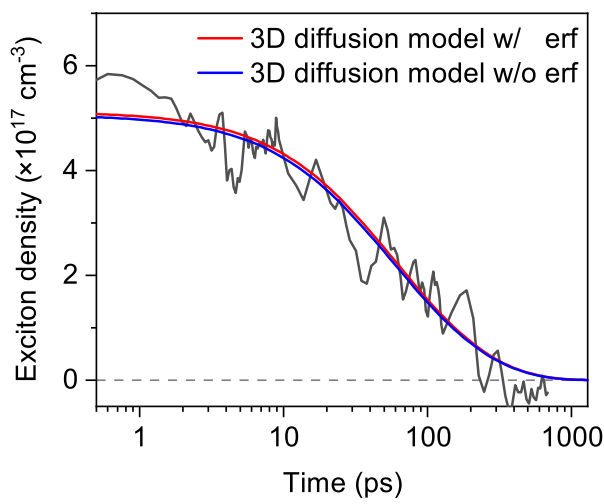

Figure 2. Singlet exciton decay kinetics for the 70-nm-thick Y6 film monitored at $970 \mathrm{~nm}$. The excitation wavelength was $800 \mathrm{~nm}$ with a fluence of $1.4 \mu \mathrm{J} \mathrm{cm}{ }^{-2}$. The red and blue lines represent the best fits obtained using the 3D diffusion model with and without the error function term, respectively.

Triplet excited states of $Y 6$ in the solid state. PIA attributed to triplet excitons generated through intersystem crossing (ISC) was clearly observed for Y6 in a CF solution (Figures S3 and S4). In contrast, as shown in Figures 1 and S6, long-lived PIA was not clearly observed for the pristine 
Y6 film, most likely due to the lower signal-to-noise ratio achieved by keeping the excitation fluence as low as possible to reduce the contribution of SSA. Therefore, we sensitized the system using Pt(II)octaethylporphyrin (PtOEP), a well-known triplet sensitizer. ${ }^{33}$ Figure 3 a shows the TA spectrum of a Y6/PtOEP blend film $(7: 1 \mathrm{w} / \mathrm{w})$ monitored at $1 \mathrm{~ns}$ after photoexcitation at $530 \mathrm{~nm}$, which reveals a large PIA band at $\sim 1400 \mathrm{~nm}$ similar to that of the Y6 triplet excitons observed in the CF solution. Thus, this band is assigned to the $\mathrm{T}_{1}-\mathrm{T}_{\mathrm{n}}$ absorption of $\mathrm{Y} 6$ in the solid state generated through triplet energy transfer from PtOEP. In the solid state, triplet excitons decayed considerably faster than that in the CF solution on the microsecond time scale (Figure S5); instead, a large negative signal was observed in the $<1200 \mathrm{~nm}$ region. This negative signal is attributable to the delayed fluorescence from Y6 following TTA, which is suggestive of fast diffusion of Y6 triplet excitons in the solid state. The fast triplet as well as singlet excitons diffusion are supposed to be related to the low level of energetic disorder in the Y6 crystalline domains.

To determine the energy level of the $\mathrm{T}_{1}$ state $E_{\mathrm{T} 1}$, we acquired the PL spectra of a mixed solution of Y6 and rubrene after selectively excitating the lower-bandgap Y6. Figure $3 b$ shows the PL spectra of a mixed solution of $\mathrm{Y} 6(1.5 \mu \mathrm{M})$ and rubrene $(300 \mu \mathrm{M})$. We observed an emission at $\sim 560 \mathrm{~nm}$, which is shorter than the excitation wavelength. As this emission band coincides with rubrene fluorescence, ${ }^{34}$ we attribute it to the upconversion (UC) emission from rubrene. Upon photoexcitation of Y6, triplet excitons are generated through ISC. Then, triplet energy transfer from Y6 to rubrene occurs to form rubrene triplet excitons that diffuse randomly and undergo TTA when they encounter one another, resulting in the generation of rubrene singlet excitons with energy higher than the excitation source. The rubrene singlet excitons decay rapidly to the ground state by emitting upconverted photons; i.e., UC emission. $E_{\mathrm{T} 1}$ of rubrene is $1.14 \mathrm{eV} ;{ }^{34}$ thus, $E_{\mathrm{T} 1}$ of Y6 is higher than $1.14 \mathrm{eV}$. To confirm that this estimation is reliable, we carried out density 
functional theory (DFT) calculations for an isolated Y6 molecule, in which the alkyl side chains were shortened to reduce the computational effort (see the Supporting Information for details). Figure $3 \mathrm{c}$ shows the natural transition orbital $(\mathrm{NTO})^{35}$ pairs of $\mathrm{Y} 6$ in the optimized $\mathrm{T}_{1}$ geometry obtained by time-dependent (TD)-DFT calculations. $E_{\mathrm{T} 1}$ was calculated to be $1.26 \mathrm{eV}$ from the
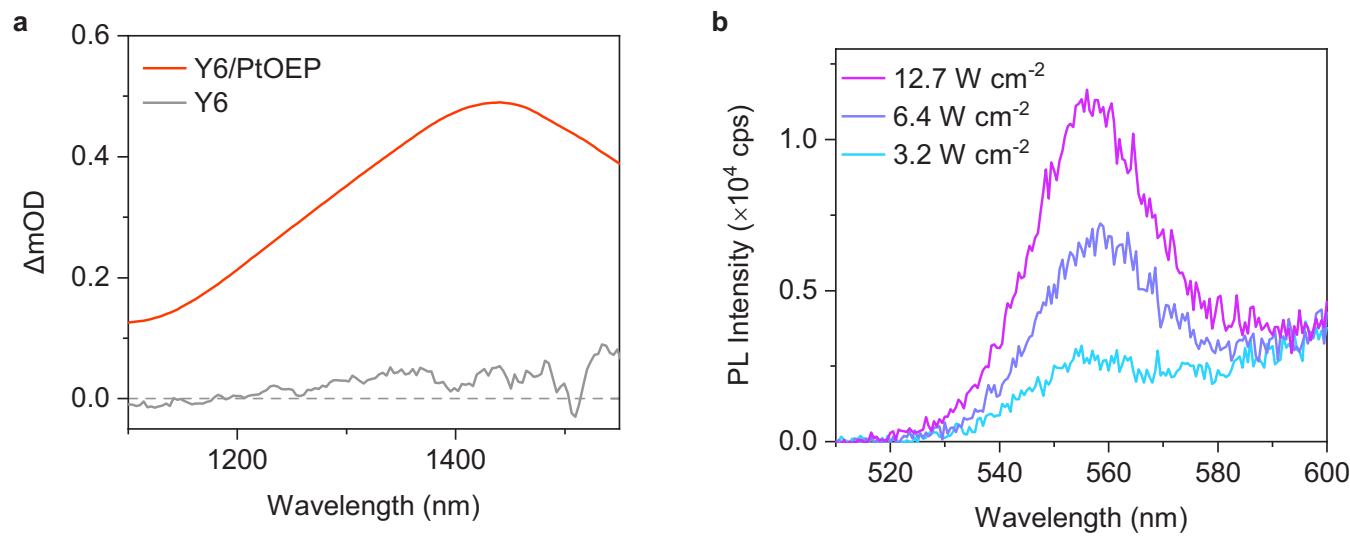

c
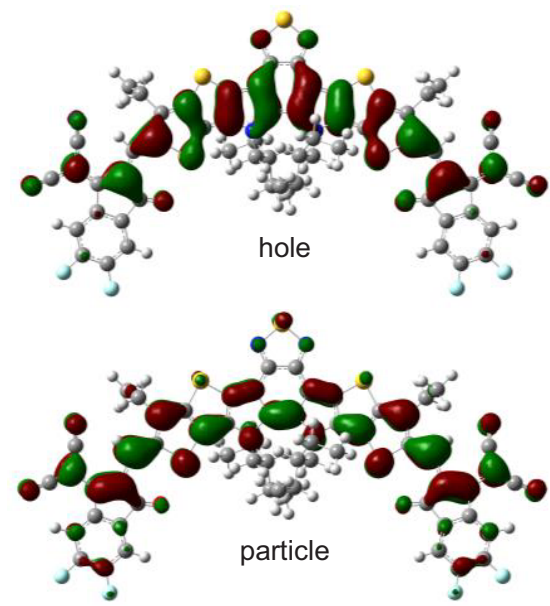

d

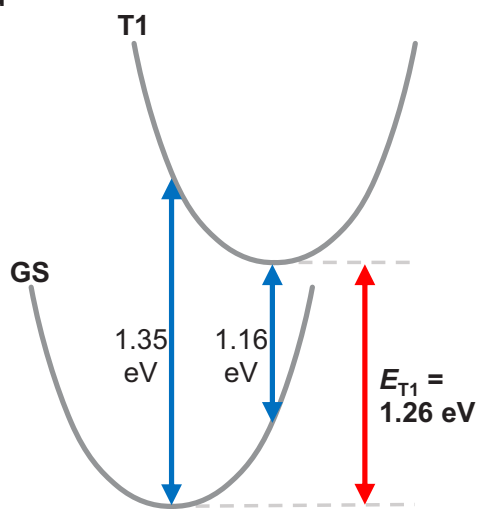

Figure 3. (a) TA spectra of a Y6/PtOEP blend $(7: 1 \mathrm{w} / \mathrm{w})$ film and a pristine $\mathrm{Y} 6$ film as a reference monitored at $1000 \mathrm{ps}$ after photoexcitation. The excitation wavelength was $530 \mathrm{~nm}$ with a fluence of $5.0 \mu \mathrm{J} \mathrm{cm}^{-2}$. (b) $\mathrm{UC}$ emission spectra of a Y6/rubrene mixture in a CF solution $(1.5 \mu \mathrm{M} / 300 \mu \mathrm{M})$ excited at $635 \mathrm{~nm}$. (c) NTO pairs for $\mathrm{Y} 6$ in the optimized $\mathrm{T}_{1}$ geometry obtained through TD-DFT calculations with B3LYP/6-31+G(d) within the Tamm-Dancoff approximation. (d) Schematic defining $E_{\mathrm{T} 1}$. 
average of two vertical excitation energies at the optimized $\mathrm{T}_{1}$ and ground state (GS) geometries (Figure 3d). Note that, in a previous study, UC emission from rubrene was also observed in a bilayer film consisting of rubrene and $\mathrm{Y} 6,{ }^{36}$ which indicates that $E_{\mathrm{T} 1}$ of $\mathrm{Y} 6$ in the solid state is also higher than that of rubrene and that the energy difference between the $\mathrm{S}_{1}$ and $\mathrm{T}_{1}$ states of $\mathrm{Y} 6$ in the solid state is less than $0.3 \mathrm{eV}$, which is considerably smaller than those of donor conjugated polymers (typically $0.5-0.7 \mathrm{eV}$ ). This small energy difference is probably due to the A-D-A'-D-A molecular architecture of Y6, which reduces the overlap between HOMO and the lowest unoccupied molecular orbital (LUMO), thereby lowering the exchange integral.

Ultrafast triplet generation through singlet fission following SSA. Triplet PIA is pronounced in the TA spectra of the pristine Y6 film at high excitation fluences, as shown in Figures 4a, 4b and S7. Accelerated triplet formation at high excitation fluences cannot be rationalized by ISC because ISC quantum efficiency is lower due to SSA. Therefore, we examined the relationship between the TA signal and the excitation fluence to investigate the mechanism of triplet generation at high excitation fluences. Figures $4 \mathrm{c}$ and $4 \mathrm{~d}$ show $\log -\log$ plots of the TA signal amplitudes monitored at 850 and $1400 \mathrm{~nm}$, respectively; these plots are well fitted by the following power-law equation: $\Delta \mathrm{OD} \propto I_{\mathrm{ex}}{ }^{m}$, where $I_{\mathrm{ex}}$ is the excitation fluence, and the slope $m$ depends on the time duration after photoexcitation. As shown in Figure 4e, the slope of the $850 \mathrm{~nm}$ signal (GSB) decreased to 0.5 on early times, indicating that SSA is the dominant decay channel for Y6 singlet excitons at high excitation fluences (detailed discussion is found in the Supporting Information). The slopes of the $850 \mathrm{~nm}$ signal recovered to unity after $500 \mathrm{ps}$, at which time singlet excitons had completely decayed, and triplet excitons were observed. In contrast, the slopes of the $1400 \mathrm{~nm}$ (superposition of singlets and triplets) remain almost unity. These results indicate that triplet excitons are generated through SSA at high excitation fluences. Figure 4f shows the decay kinetics of Y6 triplet 

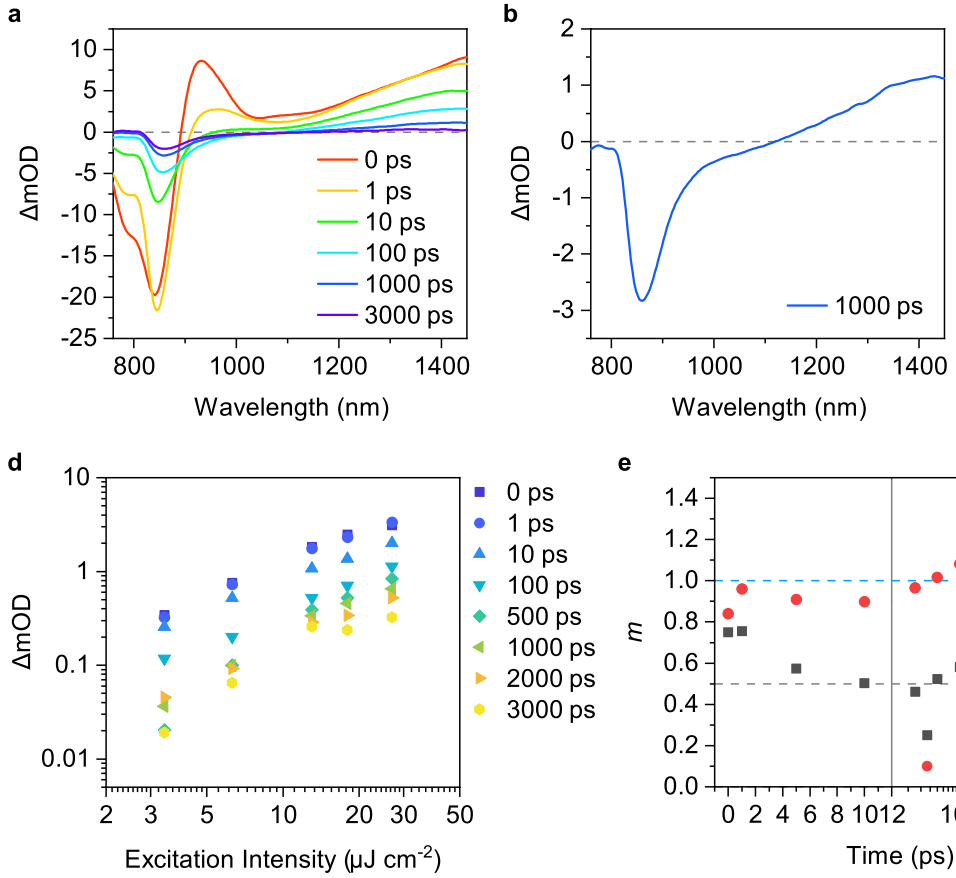
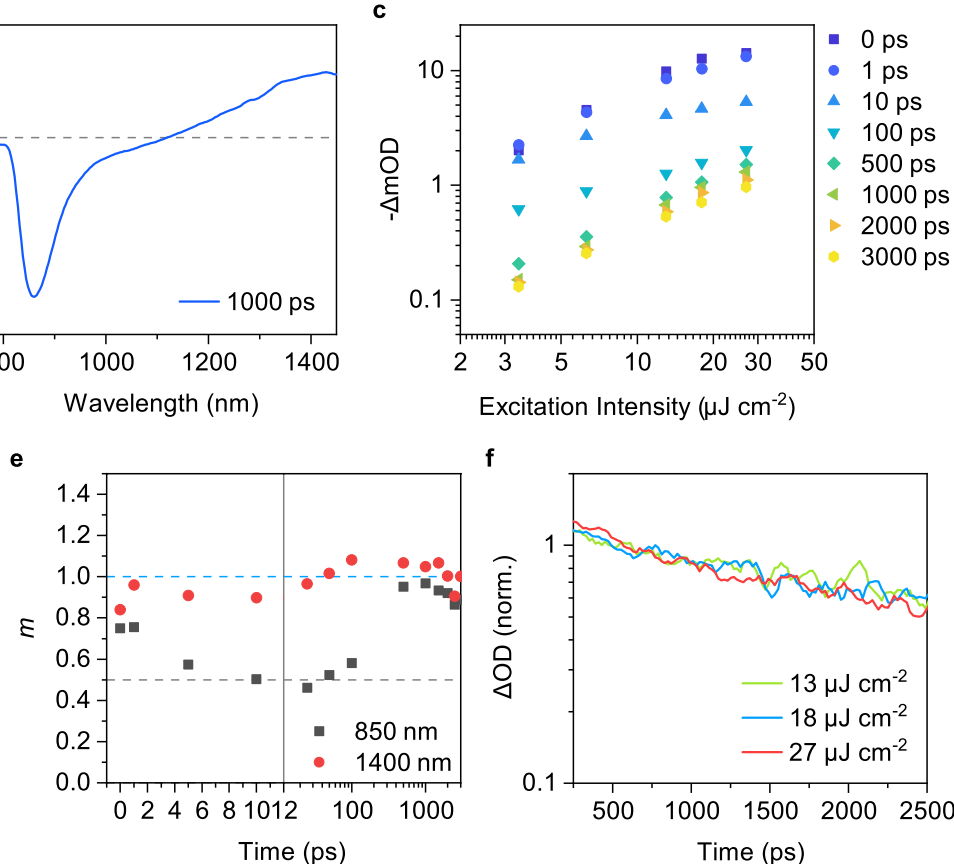

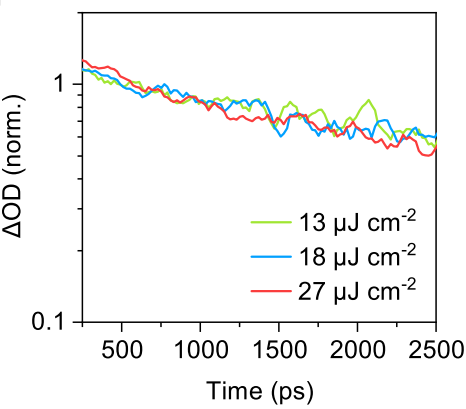

Figure 4. (a) TA spectra of the pristine Y6 film. The excitation wavelength was $800 \mathrm{~nm}$ with a fluence of $31.1 \mu \mathrm{J} \mathrm{cm}^{-2}$. (b) Enlarged TA spectra at 1000 ps. (c,d) Log-log plots of the TA signals monitored at (c) $850 \mathrm{~nm}$ and (d) $1400 \mathrm{~nm}$. (e) Time evolution of the slope $m$. (f) Excitation-fluence dependence of Y6 triplet decay.

excitons generated through SSA; these excitons decay quickly on nanosecond time scale, which is too fast to be assigned to the intrinsic Y6 triplet exciton decay as it should be on the order of microsecond (Figure S4). Interestingly, the decay dynamics of the Y6 triplet excitons generated through SSA are almost independent of the excitation fluence. Such a fluence-independent fast triplet decay has been reported for some singlet fission materials, wherein triplet pairs generated through singlet fission decay geminately before they dissociate into two free triplets. ${ }^{37,38}$ This is consistent with the observation of SE in the $900-1100 \mathrm{~nm}$ region on the nanosecond time scale, as shown in Figures $4 \mathrm{a}$ and $4 \mathrm{~b}$, which indicates that the triplet pairs recombine to the $\mathrm{S}_{1}$ state, followed by delayed fluorescence. Thus, we conclude that triplet excitons observed at high 
excitation fluences are formed through singlet fission from the $\mathrm{S}_{\mathrm{n}}$ states generated by SSA. As the $\mathrm{S}_{\mathrm{n}}$ state generated by SSA possesses twice as much energy as the $\mathrm{S}_{1}$ state, the $\mathrm{S}_{\mathrm{n}}$ state always satisfies the energetic requirement for singlet fission; i.e., $E_{\mathrm{S}}>2 E_{\mathrm{T}}{ }^{39}$ This process has been reported for anthracene for the first time, ${ }^{39}$ as well as for some conjugated polymers. ${ }^{37,38}$ This result also implies that the slow PL decay mentioned above may be a result of the geminate recombination of the triplet pairs instead of free triplet excitons because the $S_{n}$ states can also be generated after the 400-nm excitation.

In summary, we have studied the singlet and triplet excited-state dynamics of Y6 in solution and solid states. We found that singlet excitons in pristine Y6 films exhibit biphasic decay kinetics with decay constants of $\sim 220$ ps and $\sim 1200$ ps. The majority of the Y6 singlet excitons decayed with the faster $(\sim 220 \mathrm{ps})$ component, whereas the Y6 films continued to exhibit PL on the nanosecond time scale. Accordingly, we speculate that the slow PL may be induced by the $\mathrm{S}_{1}$ state repopulated through TTA of free triplet excitons or geminate recombination of triplet pairs following singlet fission. We also speculate the possible presence of highly ordered regions with a suppressed nonradiative decay rate in the pristine Y6 films. Y6 exhibits relatively fast singlet exciton diffusion with a diffusion constant of $2.1 \times 10^{-2} \mathrm{~cm}^{2} \mathrm{~s}^{-1}$, which is crucial for efficient photocurrent generation in Y6-based OSCs. Fast singlet exciton diffusion easily causes SSA at high excitation fluences. The $\mathrm{S}_{\mathrm{n}}$ states generated by SSA undergo singlet fission to form triplet pairs in competition with vibrational relaxation to the $S_{1}$ state. Although SSA-mediated singlet fission does not improve the PCE of OSCs because it generates two triplet excitons from two singlet excitons, the observation of singlet fission from the $S_{n}$ states highlights the potential application of Y6 as a photon multiplier because the $S_{n}$ states can also be generated by irradiation with UV-visible light. The small $(<0.3 \mathrm{eV})$ energy difference between the $\mathrm{S}_{1}$ and $\mathrm{T}_{1}$ states of $\mathrm{Y} 6$ 
in the solid state is another important property. Because the energy level of the $T_{1}$ state is a critical factor governing the bimolecular charge recombination rate in OSCs, the small $\mathrm{S}_{1}-\mathrm{T}_{1}$ energy difference is the key to further improve Y6-based OSCs. 
Notes

The authors declare no competing financial interests.

ORCID

Yasunari Tamai: 0000-0002-3074-0208

Hideo Ohkita: 0000-0002-7403-3492

\section{ACKNOWLEDGMENTS}

This study was partly supported by the JST PRESTO program Grant Number JPMJPR1874, JSPS KAKENHI Grant-in-Aid for Scientific Research (B) No. 21H02012, The Murata Science Foundation, The Sumitomo Foundation, and Ogasawara Toshiaki Memorial Foundation.

\section{Supporting Information}

Supporting Information is available free of charge via the Internet at http://pubs.acs.org.

Experimental details, chemical structure of Y6, steady-state absorption and PL spectra, PL decay, assignment of Y6 singlet excitons, assignment of Y6 triplet excitons, TA spectra of the 240-nmthick Y6 film, TA spectra at a high excitation fluence, SSA model, details of excitation-fluence dependence 


\section{References}

1. Liu, Q.; Jiang, Y.; Jin, K.; Qin, J.; Xu, J.; Li, W.; Xiong, J.; Liu, J.; Xiao, Z.; Sun, K.; Yang, S.;

Zhang, X.; Ding, L. 18\% Efficiency Organic Solar Cells. Sci. Bull. 2020, 65, 272-275.

2. Liu, F.; Zhou, L.; Liu, W.; Zhou, Z.; Yue, Q.; Zheng, W.; Sun, R.; Liu, W.; Xu, S.; Fan, H.;

Feng, L.; Yi, Y.; Zhang, W.; Zhu, X. Organic Solar Cells with 18\% Efficiency Enabled by an Alloy Acceptor: A Two-in-One Strategy. Adv. Mater. 2021, 33, 2100830.

3. Zhang, M.; Zhu, L.; Zhou, G.; Hao, T.; Qiu, C.; Zhao, Z.; Hu, Q.; Larson, B. W.; Zhu, H.; Ma, Z.; Tang, Z.; Feng, W.; Zhang, Y.; Russell, T. P.; Liu, F. Single-Layered Organic Photovoltaics with Double Cascading Charge Transport Pathways: 18\% Efficiencies. Nat. Commun. 2021, 12, 309.

4. Tamai, Y.; Fan, Y.; Kim, V. O.; Ziabrev, K.; Rao, A.; Barlow, S.; Marder, S. R.; Friend, R. H.; Menke, S. M. Ultrafast Long-Range Charge Separation in Nonfullerene Organic Solar Cells. ACS Nano 2017, 11, 12473-12481.

5. Hou, J.; Inganäs, O.; Friend, R. H.; Gao, F. Organic Solar Cells Based on Non-Fullerene Acceptors. Nat. Mater. 2018, 17, 119-128.

6. Zhang, G. Y.; Zhao, J. B.; Chow, P. C. Y.; Jiang, K.; Zhang, J. Q.; Zhu, Z. L.; Zhang, J.; Huang, F.; Yan, H. Nonfullerene Acceptor Molecules for Bulk Heterojunction Organic Solar Cells. Chem. Rev. 2018, 118, 3447-3507.

7. Wadsworth, A.; Moser, M.; Marks, A.; Little, M. S.; Gasparini, N.; Brabec, C. J.; Baran, D.; McCulloch, I. Critical Review of the Molecular Design Progress in Non-Fullerene Electron Acceptors Towards Commercially Viable Organic Solar Cells. Chem. Soc. Rev. 2019, 48, 15961625. 
8. Cui, Y.; Yao, H. F.; Zhang, J. Q.; Zhang, T.; Wang, Y. M.; Hong, L.; Xian, K. H.; Xu, B. W.; Zhang, S. Q.; Peng, J.; Wei, Z. X.; Gao, F.; Hou, J. H. Over 16\% Efficiency Organic Photovoltaic Cells Enabled by a Chlorinated Acceptor with Increased Open-Circuit Voltages. Nat. Commun. 2019, 10,8 .

9. Yuan, J.; Zhang, Y. Q.; Zhou, L. Y.; Zhang, G. C.; Yip, H. L.; Lau, T. K.; Lu, X. H.; Zhu, C.; Peng, H. J.; Johnson, P. A.; Leclerc, M.; Cao, Y.; Ulanski, J.; Li, Y. F.; Zou, Y. P. Single-Junction Organic Solar Cell with over 15\% Efficiency Using Fused-Ring Acceptor with Electron-Deficient Core. Joule 2019, 3, 1140-1151.

10. Saito, T.; Natsuda, S.-i.; Imakita, K.; Tamai, Y.; Ohkita, H. Role of Energy Offset in Nonradiative Voltage Loss in Organic Solar Cells. Sol. RRL 2020, 4, 2000255.

11. Umeyama, T.; Igarashi, K.; Sasada, D.; Tamai, Y.; Ishida, K.; Koganezawa, T.; Ohtani, S.; Tanaka, K.; Ohkita, H.; Imahori, H. Efficient Light-Harvesting, Energy Migration, and Charge Transfer by Nanographene-Based Nonfullerene Small-Molecule Acceptors Exhibiting Unusually Long Excited-State Lifetime in the Film State. Chem. Sci. 2020, 11, 3250-3257.

12. Armin, A.; Li, W.; Sandberg, O. J.; Xiao, Z.; Ding, L.; Nelson, J.; Neher, D.; Vandewal, K.; Shoaee, S.; Wang, T.; Ade, H.; Heumüller, T.; Brabec, C.; Meredith, P. A History and Perspective of Non-Fullerene Electron Acceptors for Organic Solar Cells. Adv. Energy Mater. 2021, 11, 2003570.

13. Karki, A.; Vollbrecht, J.; Dixon, A. L.; Schopp, N.; Schrock, M.; Reddy, G. N. M.; Nguyen, T.-Q. Understanding the High Performance of over 15\% Efficiency in Single-Junction Bulk Heterojunction Organic Solar Cells. Adv. Mater. 2019, 31, 1903868. 
14. Hosseini, S. M.; Tokmoldin, N.; Lee, Y. W.; Zou, Y.; Woo, H. Y.; Neher, D.; Shoaee, S. Putting Order into PM6:Y6 Solar Cells to Reduce the Langevin Recombination in $400 \mathrm{~nm}$ Thick Junction. Sol. RRL 2020, 4, 2000498.

15. Tokmoldin, N.; Hosseini, S. M.; Raoufi, M.; Phuong, L. Q.; Sandberg, O. J.; Guan, H.; Zou, Y.; Neher, D.; Shoaee, S. Extraordinarily Long Diffusion Length in PM6:Y6 Organic Solar Cells. J. Mater. Chem. A 2020, 8, 7854-7860.

16. Perdigón-Toro, L.; Zhang, H.; Markina, A.; Yuan, J.; Hosseini, S. M.; Wolff, C. M.; Zuo, G.;

Stolterfoht, M.; Zou, Y.; Gao, F.; Andrienko, D.; Shoaee, S.; Neher, D. Barrierless Free Charge Generation in the High-Performance PM6:Y6 Bulk Heterojunction Non-Fullerene Solar Cell. Adv. Mater. 2020, 32, 1906763.

17. Zhu, W.; Spencer, A. P.; Mukherjee, S.; Alzola, J. M.; Sangwan, V. K.; Amsterdam, S. H.; Swick, S. M.; Jones, L. O.; Heiber, M. C.; Herzing, A. A.; Li, G.; Stern, C. L.; DeLongchamp, D. M.; Kohlstedt, K. L.; Hersam, M. C.; Schatz, G. C.; Wasielewski, M. R.; Chen, L. X.; Facchetti, A.; Marks, T. J. Crystallography, Morphology, Electronic Structure, and Transport in NonFullerene/Non-Indacenodithienothiophene Polymer:Y6 Solar Cells. J. Am. Chem. Soc. 2020, 142, $14532-14547$.

18. Zhang, G.; Chen, X.-K.; Xiao, J.; Chow, P. C. Y.; Ren, M.; Kupgan, G.; Jiao, X.; Chan, C. C. S.; Du, X.; Xia, R.; Chen, Z.; Yuan, J.; Zhang, Y.; Zhang, S.; Liu, Y.; Zou, Y.; Yan, H.; Wong, K. S.; Coropceanu, V.; Li, N.; Brabec, C. J.; Bredas, J.-L.; Yip, H.-L.; Cao, Y. Delocalization of Exciton and Electron Wavefunction in Non-Fullerene Acceptor Molecules Enables Efficient Organic Solar Cells. Nat. Commun. 2020, 11, 3943.

19. Tokmoldin, N.; Vollbrecht, J.; Hosseini, S. M.; Sun, B.; Perdigón-Toro, L.; Woo, H. Y.; Zou, Y.; Neher, D.; Shoaee, S. Explaining the Fill-Factor and Photocurrent Losses of Nonfullerene 
Acceptor-Based Solar Cells by Probing the Long-Range Charge Carrier Diffusion and Drift Lengths. Adv. Energy Mater. 2021, 11, 2100804.

20. Wu, J.; Lee, J.; Chin, Y.-C.; Yao, H.; Cha, H.; Luke, J.; Hou, J.; Kim, J.-S.; Durrant, J. R. Exceptionally Low Charge Trapping Enables Highly Efficient Organic Bulk Heterojunction Solar Cells. Energy Environ. Sci. 2020, 13, 2422-2430.

21. Firdaus, Y.; Le Corre, V. M.; Karuthedath, S.; Liu, W.; Markina, A.; Huang, W.; Chattopadhyay, S.; Nahid, M. M.; Nugraha, M. I.; Lin, Y.; Seitkhan, A.; Basu, A.; Zhang, W.; McCulloch, I.; Ade, H.; Labram, J.; Laquai, F.; Andrienko, D.; Koster, L. J. A.; Anthopoulos, T. D. Long-Range Exciton Diffusion in Molecular Non-Fullerene Acceptors. Nat. Commun. 2020, $11,5220$.

22. Classen, A.; Chochos, C. L.; Lüer, L.; Gregoriou, V. G.; Wortmann, J.; Osvet, A.; Forberich, K.; McCulloch, I.; Heumüller, T.; Brabec, C. J. The Role of Exciton Lifetime for Charge Generation in Organic Solar Cells at Negligible Energy-Level Offsets. Nat. Energy 2020, 5, 711719.

23. Zhan, L.; Li, S.; Lau, T.-K.; Cui, Y.; Lu, X.; Shi, M.; Li, C.-Z.; Li, H.; Hou, J.; Chen, H. Over 17\% Efficiency Ternary Organic Solar Cells Enabled by Two Non-Fullerene Acceptors Working in an Alloy-Like Model. Energy Environ. Sci. 2020, 13, 635-645.

24. Wen, G.; Hu, R.; Su, X.; Chen, Z.; Zhang, C.; Peng, J.; Zou, X.; He, X.; Dong, G.; Zhang, W. Excited-State Properties of Y-Series Small Molecule Semiconductors. Dyes and Pigments 2021, $192,109431$.

25. Liang, S.; Li, S.; Zhang, Y.; Li, T.; Zhou, H.; Jin, F.; Sheng, C.; Ni, G.; Yuan, J.; Ma, W.; Zhao, H. Efficient Hole Transfer via Delocalized Excited State in Small Molecular Acceptor: A 
Comparative Study on Photodynamics of PM6:Y6 and PM6:ITIC Organic Photovoltaic Blends. Adv. Funct. Mater. 2021, 2102764, DOI: 10.1002/adfm.202102764.

26. Wang, R.; Xu, J.; Fu, L.; Zhang, C.; Li, Q.; Yao, J.; Li, X.; Sun, C.; Zhang, Z.-G.; Wang, X.; Li, Y.; Ma, J.; Xiao, M. Nonradiative Triplet Loss Suppressed in Organic Photovoltaic Blends with Fluoridated Nonfullerene Acceptors. J. Am. Chem. Soc. 2021, 143, 4359-4366.

27. Chow, P. C. Y.; Chan, C. C. S.; Ma, C.; Zou, X.; Yan, H.; Wong, K. S. Factors That Prevent Spin-Triplet Recombination in Non-Fullerene Organic Photovoltaics. J. Phys. Chem. Lett. 2021, $12,5045-5051$.

28. Karki, A.; Vollbrecht, J.; Gillett, A. J.; Xiao, S. S.; Yang, Y.; Peng, Z.; Schopp, N.; Dixon, A. L.; Yoon, S.; Schrock, M.; Ade, H.; Reddy, G. N. M.; Friend, R. H.; Nguyen, T.-Q. The Role of Bulk and Interfacial Morphology in Charge Generation, Recombination, and Extraction in NonFullerene Acceptor Organic Solar Cells. Energy Environ. Sci. 2020, 13, 3679-3692.

29. Tamai, Y.; Matsuura, Y.; Ohkita, H.; Benten, H.; Ito, S. One-Dimensional Singlet Exciton Diffusion in Poly(3-hexylthiophene) Crystalline Domains. J. Phys. Chem. Lett. 2014, 5, 399-403. 30. Murata, Y.; Takeyama, T.; Sakamoto, Y.; Yamaguchi, K.; Tamai, Y.; Ohkita, H. TwoDimensional Exciton Diffusion in an HJ-Aggregate of Naphthobisoxadiazole-Based Copolymer Films. J. Phys. Chem. C 2020, 124, 13063-13070.

31. Tamai, Y.; Ohkita, H.; Benten, H.; Ito, S. Exciton Diffusion in Conjugated Polymers: From Fundamental Understanding to Improvement in Photovoltaic Conversion Efficiency. J. Phys. Chem. Lett. 2015, 6, 3417-3428.

32. Chandrabose, S.; Chen, K.; Barker, A. J.; Sutton, J. J.; Prasad, S. K. K.; Zhu, J.; Zhou, J.; Gordon, K. C.; Xie, Z.; Zhan, X.; Hodgkiss, J. M. High Exciton Diffusion Coefficients in Fused Ring Electron Acceptor Films. J. Am. Chem. Soc. 2019, 141, 6922-6929. 
33. Sakamoto, Y.; Tamai, Y.; Ohkita, H. Sensitizer-Host-Annihilator Ternary-Cascaded Triplet Energy Landscape for Efficient Photon Upconversion in the Solid State. J Chem Phys 2020, 153, 161102.

34. Ma, L.; Zhang, K.; Kloc, C.; Sun, H.; Michel-Beyerle, M. E.; Gurzadyan, G. G. Singlet Fission in Rubrene Single Crystal: Direct Observation by Femtosecond Pump-Probe Spectroscopy. Phys. Chem. Chem. Phys. 2012, 14, 8307-8312.

35. Martin, R. L. Natural Transition Orbitals. J. Chem. Phys. 2003, 118, 4775-4777.

36. Izawa, S.; Hiramoto, M. Efficient Solid-State Photon Upconversion Enabled by Spin Inversion at Organic Semiconductor Interface. ChemRxiv, DOI: 10.26434/chemrxiv.14184830.v1.

37. Tamai, Y.; Ohkita, H.; Benten, H.; Ito, S. Singlet Fission in Poly(9,9-di- $n$-octylfluorene) Films. J. Phys. Chem. C 2013, 117, 10277-10284.

38. Kasai, Y.; Tamai, Y.; Ohkita, H.; Benten, H.; Ito, S. Ultrafast Singlet Fission in a Push-Pull Low-Bandgap Polymer Film. J Am Chem Soc 2015, 137, 15980-15983.

39. Smith, M. B.; Michl, J. Singlet Fission. Chem. Rev. 2010, 110, 6891-6936. 